\title{
Beruf Haustier Im Fotoatelier
}

Erschienen in: Mensch und Tier

Von: Stefanie Grebe

Angebote für Tierfotografie oder ,künstlerische Verewigungen der Gefährten („Dein Haustier als Portrait") sind im Internet in großer Anzahl anzutreffen. Vorläufer dieses Booms, die im Prozess der Erschließung und Digitalisierung des Bestandes eines Altenessener Fotostudios zum Vorschein kamen, dienen im Folgenden als Ausgangspunkt einer Reflexion darüber, welche Perspektiven und Blickwechsel das Verhältnis von Mensch und Tier auch in fotografischen Altagspraktiken mitbestimmen.

\section{Domestizierung}

Ein Ziel der Domestizierung von Tieren bestand in ihrer wirtschaftlichen Nutzung für den Menschen, vor allem dienten und dienen Tiere als Lieferanten für Nahrungsmittel, Gebrauchsgüter, als Transportmittel oder Arbeitshilfe. Die Möglichkeiten von Arbeitseinsätzen waren und sind dabei sehr vielfältig: Tiere können bei der Jagd helfen, Botschaften transportieren, die Herde, das Haus und den Hof bewachen, warnen und Feinde angreifen, bestimmte Stoffe erspüren, sie können Lasten ziehen oder tragen oder als Blindenhund Menschen führen. Neuerdings arbeiten sie sogar nicht nur im physischen, sondern auch im psychotherapeutischen Einsatz.

Bei allen Arbeitseinsätzen von Pferden, Hunden, Rindern, Elefanten, Ziegen bestehen unterschiedliche Wechselwirkungen zwischen Mensch und Tier; in der Regel ,spannen“ die Menschen die Tiere als günstiges und effektives Hilfsmittel im wahrsten Sinne des Wortes ,ein'. Es besteht nur selten ein gleichberechtigtes Miteinander; die Abhängigkeit des Menschen vom Tier ist aber dennoch groß. Wenn dereinst eine andere Tier-Ethik den Diskurs dominieren wird, könnte es sogar sein, dass das Halten von Nutz- und Haustieren in Verruf geraten wird. Haustier sein ist ein besonderer Beruf, in dessen Zentrum Macht und Liebe stehen.

\section{Macht und Liebe}

Tiere lieben uns und wir lieben Tiere. Wir lieben sie, vielleicht weil wir uns in innen auf einer früheren stammesgeschichtlichen Entwicklungsstufe wiederfinden, und wir beneiden sie um ihre Gegenwärtigkeit und Unschuld. Tiere sind unsere Gefährten: als Haustiere, als Freunde, Seelentröster oder Partnerersatz. Die Abwesenheit realer Tiere wurde und wird gelindert durch Spielzeuge wie etwa Schaukelpferd und Teddybär oder elektronische Gefährten wie das Tamagotchi. Tierliebe ist in sich widersprüchlich, denn 
sie umfasst Verhältnisse von Abhängigkeit und Ausnutzung, Schutz und Wertschätzung . Obwohl gerade Haustiere wie Hunde den Besitzer*innen bedingungslose Liebe entgegenbringen, behalten die Menschen die Kontrolle und Macht über sie. Auch der Grundkonflikt „wir lieben und wir essen sie“ bleibt ungelöst.

Um uns den Tieren anzunähern, scheint die Vermenschlichung der meistgeliebten tierischen Gefährten unwiderstehlich: Das Tier wird zur Comic-, zur Zeichentrick- oder Fabelfigur und verkörpert vor allem menschliche Eigenschaften. Tiere werden verkleidet, zu Kunststücken dressiert oder im Fotostudio porträtiert.

\section{Im Fotostudio von Helga Hethey}

Ins „Fotoatelier Helga Hethey“ in Essen-Altenessen, das die Fotografen-Meisterin von 1974 bis 1989 allein unterhielt, kamen täglich Menschen, um sich zu besonderen Gelegenheiten wie Hochzeit, Konfirmation und Kommunion fürs Album oder für ein Passfoto ablichten zu lassen. Das Studio existierte bereits seit 1936 als Fotoatelier von Carl Lenz (1899-1968) auf der Altenessener Str. 354 als „Foto Lenz“. Die Fotografin Helga Hethey trat 1952 in das im Stadtteil beliebte und renommierte Atelier ein. Die 1950er und 1960er Jahre bescherten dem Atelier gute Zeiten, es lebten ca. 60.000 Menschen im Stadtteil, die vor allem durch die sieben Zechen in Vogelheim und Altenessen ernährt wurden. Auch die als Gastarbeiter gekommenen

Arbeitsmigrant*innen suchten das Atelier für ein Einzel- oder Familienporträt auf, um ihre Familien in der Ferne von ihrem

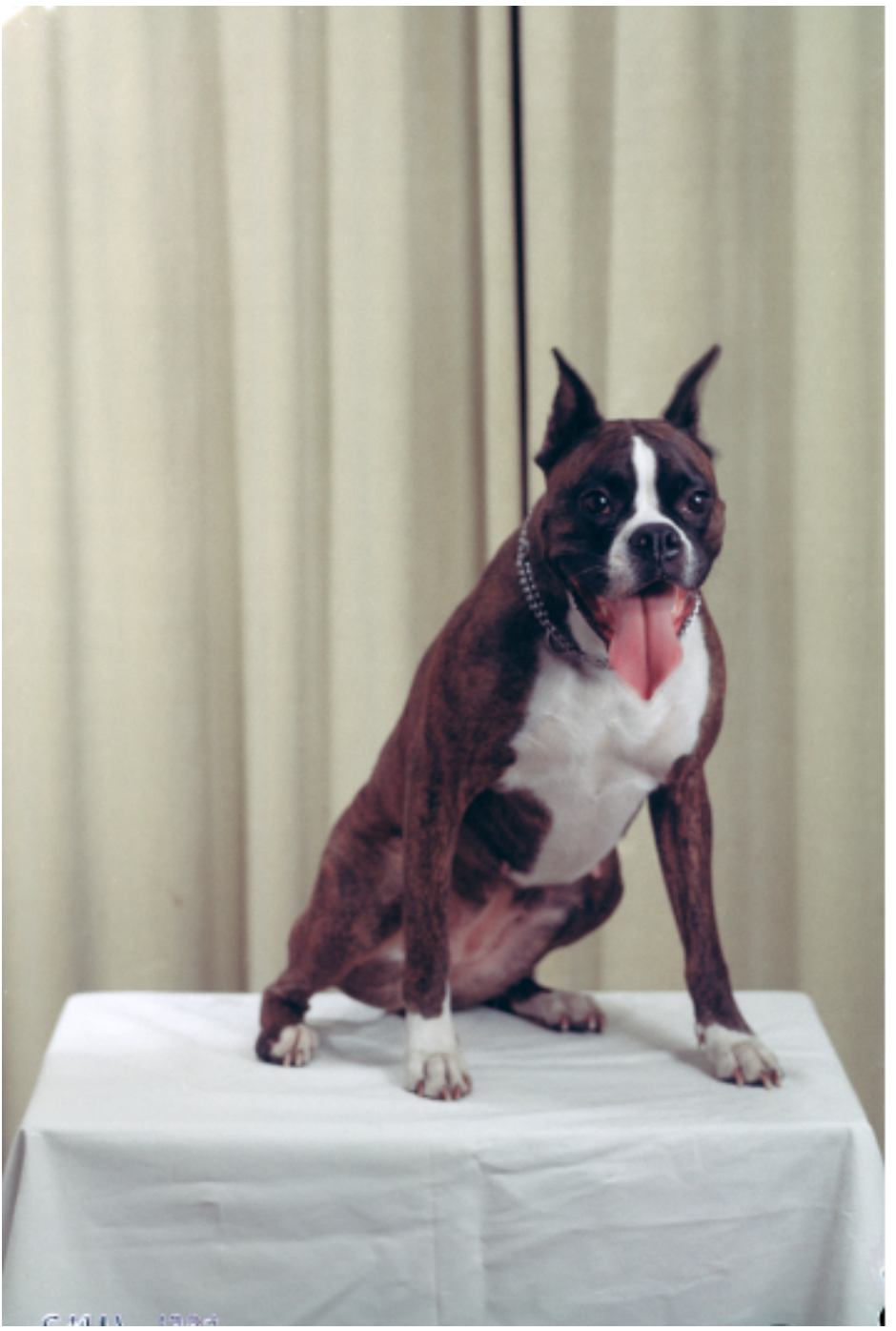

Abb. 1: Essen 1975-1979, Helga Hethey/Fotoarchiv Ruhr Museum gelungenen Leben zu überzeugen.

Ein Porträt kostete ca. 110 DM, Retusche inklusive. Diese Studioporträts vermitteln überdeutlich die Zeichen der Zeit, die Haar- und Kleider- und auch die fotografischen Darstellungsmoden. Wie bei aller Studio-Porträtfotografie sollten die Abgebildeten so dargestellt werden, dass ihr Selbstbild mit dem fotografierten Fremdbild in Einklang zu 
bringen war und sie ihr Bild auch kaufen wollten. Das Ergebnis musste also sowohl realitätsgetreu als auch idealisierend sein. Dieser Spagat gelang Helga Hethey, die ein Gespür für die Eigenheiten ihrer Kund*innen hatte.

Der Hauptauftraggeber des Ateliers war die ortsansässige Industrie, die Dokumentationen und Werbung benötigte. Nach dem Tod von Lenz und der Übernahme durch Hethey gingen die Einnahmen aus unterschiedlichen Gründen kontinuierlich zurück: Passfotos wurden durch Automaten erledigt, Familienfeste mit Video festgehalten und Amateuraufnahmen ersetzten das professionelle Porträt. Mit dem drohenden Abriss des Geschäftshauses gab Helga Hethey ihr Geschäft 1989 auf. Das Fotoarchiv des Ruhrlandmuseums erhielt den fotografischen Bestand aus der Nachkriegszeit mit ca. 30.000 Negativen aller Art. Helga Hethey, geboren als Helga Overbeck 1926 in Essen, verstarb 2019.

In ihrem Atelier entstanden Ganzkörper-Aufnahmen, Halb-, Kopf-, Einzelporträts oder Gruppenbildnisse. Da keine Auftragsbücher überliefert sind, kann der Anlass der Aufnahme nicht mehr eindeutig zurückverfolgt werden, es sei denn, es handelt sich um eine Feierlichkeit, bei der die Kleidung Aufschluss geben kann. Auf den meisten Schwarzweiß- und Farb-Großbild-Negativen aus dem Konvolut der Porträts der Altenessener Kundschaft, die einzeln in Pergamintaschen aufbewahrt werden, steht der Name der Auftraggeber*innen, die Adresse und das Datum der Aufnahme. Zwischen den ca. 30.000 Negativen und 140 Vintages des Fotoateliers aus den Jahren 1950 bis1989 im Fotoarchiv des Ruhr Museums, befinden sich vereinzelte Aufnahmen von Hunden und Katzen. Diese Haustier-Aufnahmen, deren Zahl bislang noch nicht vollständig erfasst ist, sind mit dem Namen der Tier-Besitzerinnen gekennzeichnet. Der Name des abgebildeten Tieres wird nicht genannt.

Nicht geklärt ist, ob die Tierporträts im Schaufenster des Studios ausgestellt wurden, um die Kund*innen zu dieser Art des Porträts zu motivieren. Der Fund eines handcolorierten Silbergelatine-Barytprints im Format $40 \times 30 \mathrm{~cm}$, das einen Boxer vor dem selben Hintergrund wie beim hier abgebildeten Boxer zeigt, lässt dies jedoch vermuten. Die Eiweißlasurfarbe des colorierten Schwarzweißabzugs verblasste im Gegensatz zum Color-Print nicht durch UV-Licht. Für andere auf diese Art entstandenen Abzüge ist überliefert, dass sie sich im Schaufenster befanden.

Auf den gefundenen Tierporträts wurden vor allem Hunde, vereinzelt aber auch Katzen, allein oder mit ihren Besitzer*innen abgebildet. Deutlich spürbar ist, wie viel Mühe sich die Fotografin gibt, die passenden Farbharmonien durch die Wahl des Unter- und Hintergrunds für die Tiere zu finden und diese auch zu einer würdigen oder auch angemessenen Pose - vermutlich mit Unterstützung der Besitzer*innen - zu bewegen. Dass Tierbesitzer*innen ihre Lieblinge abgebildet sehen wollen, hat eine lange Tradition in der Bildenden Kunst. Sogar in Zeiten der digitalen Fotografie werden Haustiere auch heute noch gemalt. Anders als in der Tierfotografie, die wildlebende Tiere im Habitat darstellt (wildlife photography), entsteht das Studioportrait des Haustiers in einem anderen Kontext, der auch andere Gesten des Zeigens und Posierens hervorbringt.

\section{Bildbeschreibungen}


Ein Hund sitzt auf einer

Tischdecke, die über einem

kleinem Tisch liegt und schaut in

Richtung der Kamera, leicht an inr

vorbei auf etwas oder jemanden, der nicht sichtbar ist. Die

Tischdecke ist aus weißer

Baumwolle, der Vorhang im

Hintergrund lindgrün. Der Hund ist ein Boxer, mit einem weißen Latz und Fleck im Gesicht, sein

Halsband ist aus Metall. Das linke Ohr hat eine Bewegungsunschärfe erzeugt. Auf der anderen

Fotografie ist die Tischdecke aus einem gelben Wollgewebe und der Vorhang im Hintergrund rot. Der Welpe in dieser Szene ist schwarzweiß gescheckt. Sein Halsband ist aus braunem Leder und noch zu groß für ihn. Auf der dritten Fotografie sitzen zwei junge Frauen rechts und links neben einem weißen Pudel, der von einer der beiden Frauen gehalten wird; alle drei blicken in die Kamera.

Der Tisch für den Hund ist von der gelben Wolldecke bedeckt, im

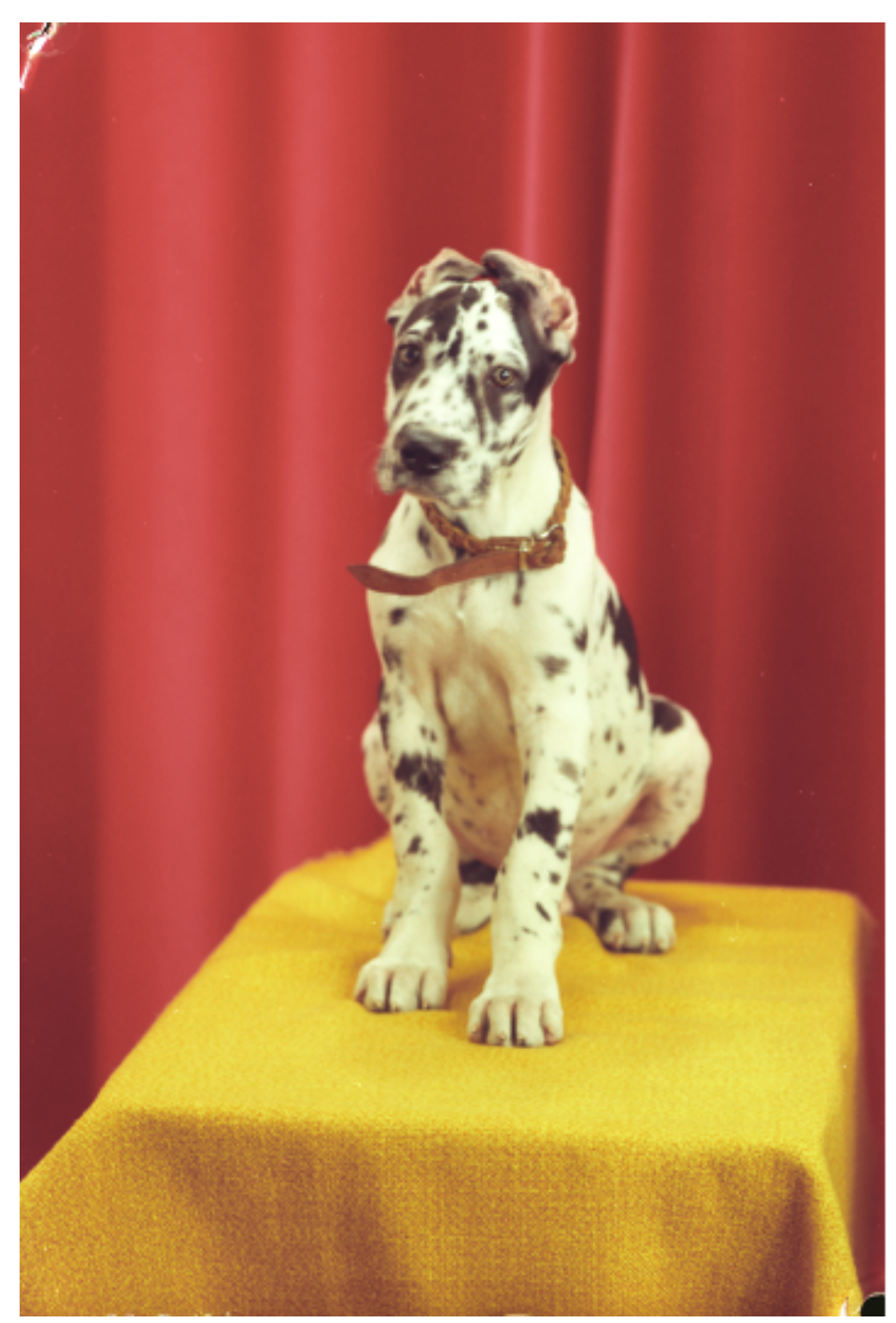

Abb. 2: Essen 1975-1979, Helga Hethey/Fotoarchiv Ruhr Museum Hintergrund ein bläulicher

Vorhang. Alle drei Modelle tragen ein rotes Kleidungsstück und sind frisch frisiert. Es ist Mitte bis Ende der 1970er Jahre.

Wenn solche Porträts von Haustieren im Fotoatelier geschaffen werden, steht offensichtlich das Menschliche und Domestizierte des Tiers im Vordergrund. Es wird wie ein Mensch dargestellt, als Wesen mit einem Blick, der kulturell für Persönlichkeit und Seele steht. So wirkt der Welpe unsicher und fragend, der ältere Artgenosse scheint ein gesundes Selbstbewusstsein zu besitzen, wobei der Pudel, der mit zwei jungen Frauen abgebildet wird, manierlich und aufmerksam wirkt. Zugleich wird der Hund in Ausübung seiner beruflichen Tätigkeit als Haustier gezeigt, also als Liebesobjekt, als Liebender, Therapeut, Tröster, Wächter usw.

Sind diese Zuschreibungen unserem vermenschlichenden Blick zuzuordnen, der nichts anderes kennt als menschliche Kategorien, oder sind sie ,wirklich' da? Vielleicht sollte der Fokus weniger auf der latenten Kritik der Zuschreibung menschlicher Eigenschaften auf Tiere liegen, als auf der Fähigkeit, sich in andere Lebewesen einzufühlen. Gerade der Blick spielt hier eine herausragende Rolle. Der Blick des noch mit der Situation 
unvertrauten Kleinkindes in eine Kamera kann ein ähnliches Unbehagen bereiten wie ein Hundeblick: Wir ahnen, dass es keine wirklich freiwillige Entscheidung des Kindes und Hundes zu diesem Akt gab. Geht der Hund - erzwungenermaßen ins Fotoatelier, kommt ein Bild heraus, das ein Machtgefälle illustriert und Mitgefühl provoziert.

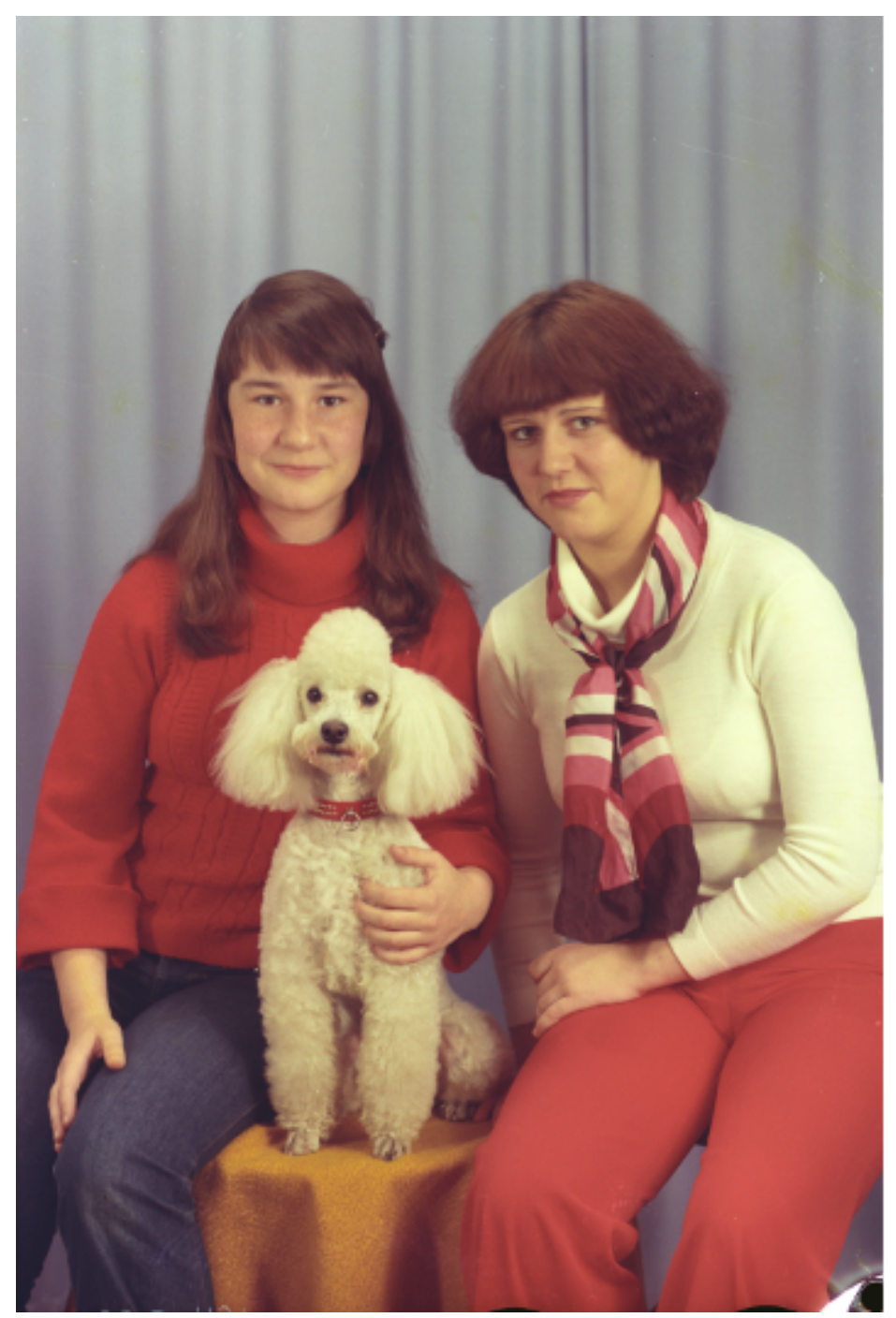

Abb. 3: Essen 1975-1979, Helga Hethey/Fotoarchiv Ruhr Museum

\section{References}

1. Borgards, Roland (2017): Die Tiere und wir, in: Schulze, Sabine; Conrad, Dennis $(\mathrm{Hg}$.): Tiere: Respekt - Harmonie - Unterwerfung, Ausstellungskatalog Museum für Kunst und Gewerbe Hamburg, München, S. 27-33.

2. Eskildsen, Ute; Lechtreck, Hans-Jürgen (Hg.) (2005): nützlich, süß und museal / das fotografierte Tier, Ausstellungskatalog Museum Folkwang Essen, Göttingen (bes. S. 124-153 und 248-297).

3. Lillge, Claudia (2018): Tiere sehen, in: Fotogeschichte. Beiträge zur Geschichte und Ästhetik der Fotografie, Heft 148, Jg. 38, Sommer 2018, S. 3-5.

4. Macho, Thomas (2020): Tiere als Individuen, in: Jaeger, Friedrich (Hg.): Menschen und Tiere. Grundlagen und Herausforderungen der Human-Animal Studies (Cultural Animal Studies, Band 9), Berlin, S. 237-249. https://doi.org/10.1007/978-3-476-05625-2_13

5. Peters, Ursula (2009): Canis, der anklopfende Hund und andere Tiere zur Erbauung, in: Germanisches Nationalmuseum (Hg.): Vom Ansehen der Tiere, Kulturgeschichtliche Spaziergänge im Germanischen Nationalmuseum, Band 11, Nürnberg, S. 190-204. 
6. Schwabenthan, Sabine; Scheels, Walter (2000): Die Seele der Tiere. Gesichter, Gefühle, Geschichten, München (bes. S. 6).

7. Sezgin, Hilal (2014): Artgerecht ist nur die Freiheit, München, S. 133-186.

8. Ullrich, Jessica (2018): Zeugenschaft und Trauerarbeit in der zeitgenössischen Tierfotografie, in: Fotogeschichte. Beiträge zur Geschichte und Ästhetik der Fotografie, Heft 148, Jg. 38, Sommer 2018, S. $47-59$ (bes. S. 50-52).

9. Weber, Andreas (2007): Alles fühlt. Mensch, Natur und die Revolution der Lebenswissenschaften. Berlin, S. 127-167.

SUGGESTED CITATION: Grebe, Stefanie: Beruf Haustier. Im Fotoatelier, in: KWIBLOG, [https://blog.kulturwissenschaften.de/beruf-haustier/], 22.03.2021

DOI: https://doi.org/10.37189/kwi-blog/20210322-0830 


\section{DuEPublico}

Duisburg-Essen Publications online

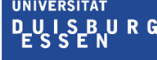

offen im Denken

$\mathbf{U b} \mid \begin{gathered}\text { universitäts } \\ \text { bibliothek }\end{gathered}$

Dieser Text wird via DuEPublico, dem Dokumenten- und Publikationsserver der Universität Duisburg-Essen, zur Verfügung gestellt. Die hier veröffentlichte Version der E-Publikation kann von einer eventuell ebenfalls veröffentlichten Verlagsversion abweichen.

DOI: $\quad$ 10.37189/kwi-blog/20210322-0830

URN: urn:nbn:de:hbz:464-20210322-100548-4 